\title{
Getting the right balance in treatment of ductal carcinoma in situ (DCIS)
}

\author{
Ian S Fentiman \\ Department of Surgical Oncology, Guy's Hospital, London, UK. \\ Received October 28, 2013; Revised December 02, 2013; Accepted December 03, 2013; Published Online December 05, 2013
}

\section{Review Article}

\begin{abstract}
As a result of mammographic detection, ductal carcinoma in situ (DCIS) is an increasing problem in breast clinics. Both histopathology and molecular profiling can identify subtypes likely to progress to invasive disease, but there is no subgroup with a zero likelihood of subsequent invasion. In patients with low/intermediate grade DCIS, if breast irradiation is not being carried out after free margins have been achieved the patient should be aware of the risks of withholding and the benefits and morbidity of adjuvant radiotherapy. Either tamoxifen or an aromatase inhibitor may be of value in those with low/intermediate ER+ve disease if radiotherapy is being withheld. For those patients with extensive or multicentric DCIS, mastectomy is the appropriate treatment. This is best combined with sentinel node biopsy and all such cases should be offered immediate reconstruction.
\end{abstract}

Keywords: Ductal carcinoma in situ; High grade; Low/intermediate grade; Recurrence; Progression; Radiotherapy; Tamoxifen; Mastectomy

\section{Introduction}

In the not so distant past, no real distinction was made between invasive breast cancer and ductal carcinoma in situ (DCIS), both being treated by mastectomy, often including an axillary clearance. Such radical surgery for DCIS became questioned as results emerged from randomised trials indicating that breast conservation was a safe and effective alternative to mastectomy for invasive breast cancer.1, 2, 3, 4,5 At the same time, as a by-product of national mammographic screening programmes, the incidence of DCIS was increasing, representing up to a quarter of screen-detected malignancy. In the US in 1975 the incidence of DCIS was 2 per 100,000 and by 1985 was 10 , rising to 22 in 1995 and 30 by $2005 .^{6}$

Was it reasonable to offer a mastectomy for a non-life threatening condition? We know now that the answer is "yes and no". For some women, as a result of incomplete excision of DCIS, progression to invasive disease will reduce life expectancy. But are we yet in a position to individualise treatment for women diagnosed with DCIS? The aim of treatment

Corresponding author: Ian S Fentiman MD DSc FRCS; Professor of Surgical Oncology, Research Oncology, $3^{\text {rd }}$ Floor Bermondsey Wing, Guy's Hospital, London SE1 9RT, UK.

Email: ian.fentiman@gstt.nhs.uk

Cite this article as:

Fentiman IS. Getting the right balance in treatment of ductal carcinoma in situ (DCIS). Int J Cancer Ther Oncol 2013;

1(2):01029. DOI: $10.14319 /$ ijcto.0102.9 is to eradicate the DCIS thereby reducing the risk of recurrence of DCIS or progression to invasive disease. DCIS is a miscellany of conditions with a spectrum of risk of malignancy and it is essential that it is not universally downgraded to being regarded as a benign precursor of with an attendant laissez faire attitude towards the management of high grade disease.

\section{Histopathology}

Randomised trials of treatment for DCIS have not only yielded evidence of relative efficacy of treatments tested but have also led to central pathological review by pathologists with a special interest in breast cancer. This has enabled the delineation of groups with differing risks of subsequent relapse or progression to invasive disease.

Pinder et al conducted a full pathological review of 1222/1694 (72 \%) of specimens from women participating in the UKCCCR/ANZ DCIS trial. ${ }^{7}$ Those pathological features associated with ipsilateral recurrence in univariate analysis included high cytonuclear grade, larger lesions, growth pattern, presence of necrosis or chronic inflammation, margin involvement or uncertainty of margin status and narrower margin width. The large group of high-grade lesions was subdivided to identify a very poor prognosis subgroup; DCIS of high cytonuclear grade, with $>50 \%$ solid architecture, 
comedo-type necrosis in $>50 \%$ of ducts. After a median follow-up of 53 months, hazard ratios for ipsilateral in situ or invasive recurrence in low, intermediate, high and very high, grade were $0.42,0.33,0.62$ and 1.00 , respectively.

Because they found little difference in ipsilateral recurrence rates between low- and intermediate-grade groups they joined these two to form a 3 tier system: low/intermediate, high and very high. Results are shown in TABLE 1 . Although the new categorisation does identify groups with different risks of DCIS relapse and progression to invasive disease, there is no group with a zero probability of invasive progression.

TABLE 1: Recurrence rates within 3 tier categorisation of DCIS from UKCCCR/ANZ DCIS trial.

\begin{tabular}{lccc}
\hline \hline Category & $\mathbf{N}$ & $\begin{array}{c}\text { DCIS } \\
\text { recurrence }\end{array}$ & $\begin{array}{c}\text { Invasive } \\
\text { progression }\end{array}$ \\
\hline Low/intermediate & 311 & $11(3.5 \%)$ & $8(2.5 \%)$ \\
High & 430 & $32(7.4 \%)$ & $14(3.3 \%)$ \\
Very high & 483 & $56(11.6 \%)$ & $32(6.6 \%)$ \\
\hline \hline
\end{tabular}

In the histopathological review of EORTC trial 10853, specimens from 863/1010 (85\%) were examined and a multivariate analysis was conducted of clinicopathological features and relapse risk after a median follow-up of 5.4 years. ${ }^{8}$ Significant variables to emerge from multivariate analysis included young age $\leq 40$ years (hazard ratio 2.14), symptomatic DCIS (HR 1.8) solid and comedo growth pattern (HR 2.6 and 2.69), margin involvement (HR 2.07) and excision without radiotherapy (HR 1.7). It is noteworthy that margin status was a more major risk factor for recurrence than avoidance of breast irradiation.

It could be argued that what is important in treatment of DCIS is the avoidance of invasive relapse rather than recurrence of DCIS since this is not life threatening. The situation is however more complex. In EORTC 10853 there was no significant relationship between the type of DCIS and risk of progression to invasion. When however the risk of distant metastasis of invasive cancer was examined it was significantly elevated in those with high grade DCIS (HR 6.57).

Esserman et al have suggested the acronym IDLE (indolent lesions of epithelial origin) to encompass abnormalities which may not be precursors of invasive cancer, such as low grade DCIS. ${ }^{9}$ This is good insofar as some patients will be saved from unnecessarily radical surgery but will be problematic if imperfectly characterised lesions are included, thereby placing them at increased risk of invasive disease.

\section{Molecular profiling}

Clark et al constructed tissue microarrays (TMA) for 188 cases of DCIS and examined expression patterns of estrogen receptor (ER), progesterone receptor (PR), HER2, EGFR, cy- tokeratin (CK) 5/6, CK14, CK17, CK18, b4-integrin, b6-integrin, p53, SMA, maspin, Bcl-2, topoisomerase IIa and P-cadherin. Hierarchical clustering analysis was undertaken to identify any natural groupings, and the findings were validated in an independent series of 75 cases. Although the intrinsic molecular subtypes could be identified in DCIS, subgroup frequency varied. In DCIS the triple negative and basal-like phenotype was very uncommon. Hierarchical cluster analysis identified three main subtypes of DCIS and the 4 main markers were ER, PR, Her2 and Bcl-2, related to conventional prognostic indicators. These subtypes ER-PR-BCL-HER2+, ER-PR-BCL-HER2-, R+PR+HER2-BCLand $\mathrm{ER}+\mathrm{PR}+\mathrm{HER} 2-\mathrm{BCL}+$ were confirmed in the validation series of DCIS cases.

Solin et al examined specimens from 327 women with pure DCIS who participated in Eastern Cooperative Oncology Group (ECOG) E5194 study. ${ }^{10}$ Those with low or intermediate grade DCIS were observed without having breast irradiation and some took tamoxifen. Oncotype DX breast cancer assay was performed to obtain a prospectively defined DCIS Score (based on 7 cancer-related genes (Ki67, STK15, Survivin, CCNB1 (cyclin B1), MYBL2, PR, GSTM1 and 5 reference genes). The continuous DCIS Score was statistically significantly associated with the risk of developing an ipsilateral breast event (IBE). For the of low, intermediate, and high DCIS risk groups the 10-year risks of developing an invasive IBE, were $3.7 \%, 12.3 \%$, and $19.2 \%$, respectively ( $P \leq$ .006). In multivariable analyses, factors significantly associated with IBE risk were DCIS Score, tumour size, and menopausal status.

\section{Need for breast irradiation}

The four major trials of treatment for DCIS had the main aim of determining whether radiotherapy was a necessary part of the treatment after excision of DCIS. ${ }^{11,12,13,14}$ All have shown the impact of breast irradiation on DCIS relapse and invasive progression. As an example, in EORTC 10853, the 10-year local relapse-free (LR) rate was $74 \%$ in the group treated with local excision (LE) alone compared with $85 \%$ in those treated by LE plus RT (log-rank $P<.0001$; hazard ratio [HR] = $0.53)$. The risks of DCIS relapse and invasive progression were reduced by $48 \%(P<.0011)$ and $42 \%(P<0065)$ respectively.

The effect of RT was homogeneous across all assessed risk factors, that is, there was no subgroup in which it could be shown that radiotherapy was redundant. ${ }^{15}$ Despite this series with long term follow-up are published in which women with completely excised low and intermediate DCIS, $<2 \mathrm{~cm}$ are treated by wide excision and no radiotherapy. In a recent publication, Wehner et al reported 205 women treated with LE alone among whom the 6 years probability of relapse was $6.6 \%{ }^{16}$, similar to that in the low/intermediate category in the UKCCCR/ANZ DCIS trial. 


\section{Adjuvant tamoxifen}

Tamoxifen has been tested in two prospective randomised trials, NSABP B $24^{17}$ and UKCCCR/ANZ DCIS. ${ }^{18}$ In B24, 1804 women with DCIS, including those with margin involvement, were treated by lumpectomy, radiotherapy, and tamoxifen $20 \mathrm{mg}$ daily for 5 years or lumpectomy, radiation therapy, and placebo. To be eligible for UKCCCR/ANZ DCIS, the 1701 participants had complete local excision of DCIS and were randomised in a $2 \times 2$ factorial manner to observation, radiotherapy, tamoxifen or radiotherapy and tamoxifen. Long-term results are shown in TABLE 2.

In B24, after a median follow-up of 163 months, the overall ipsilateral breast recurrence rate was $19 \%$ in the placebo arm and $9 \%$ in the tamoxifen group. ${ }^{19}$ There was a reduction in both DCIS relapse and invasive progression in the tamoxifen arm. Allred et al determined estrogen (ER) and progesterone receptors (PgR) in 732 (41\%) of B24 participants..$^{20}$ ER was positive in $76 \%$ of patients. Those with ER-positive DCIS who received tamoxifen had significant reduction in subsequent breast cancer at 10 years (hazard ratio [HR], 0.49; $P<0.001$ ). No significant effect was seen in ER-negative DCIS.

In UK/ANZ after 12.7 years median follow-up, tamoxifen reduced both ipsilateral DCIS relapse and contralateral events but had no impact on ipsilateral invasive progression. ${ }^{21}$ The non-concordant results from the two trials may be a reflection of age differences. In the UK/ANZ trial where most cases were derived from the screening programme $>90 \%$ were aged 50 years or older. In B24 however, most participants were younger with only $34 \%$ being aged $>50$.

Staley et al conducted a Cochrane review of post-operative tamoxifen for DCIS based on these 2 trials, totalling 3375 women. ${ }^{22}$ They concluded that tamoxifen reduced incidence of both ipsilateral and contralateral DCIS (RR 0.50). There was a non-significant trend towards more endometrial cancer in the tamoxifen group but no impact on overall mortality.

\section{Adjuvant aromatase inhibitors}

No results are yet available from randomised trials such as IBIS-2 on the effect of aromatase inhibitors in post-menopausal women with ER positive DCIS. ${ }^{23}$ Dixon et al reported that in a randomised neoadjuvant study of 206 postmenopausal women with invasive ER+ve breast 28 pre and post-treatment specimens contained ER+ve DCIS. ${ }^{24}$ Both anastrozole and letrozole significantly inhibited proliferation. There was reasonable agreement between the fall in proliferation within both the invasive and in situ components.

Chen et al conducted a neoadjuvant trial in which 9 patients with ER-positive pure DCIS diagnosed by stereotactic core biopsy were treated with letrozole, followed by excision biopsy. ${ }^{25}$ Proliferation and apoptotic markers were measured at baseline and at three months and compared with specimens from patients not given preoperative treatment. There was significant reduction of $\mathrm{PR}$, and $\mathrm{Ki67}$ as well as increase in CD68-positive cells. The authors questioned whether those responding might be able to avoid surgical intervention.

\section{Magnetic resonance imaging (MRI)}

MRI has been used particularly in women with dense breasts in an attempt to determine the extent of DCIS, in the hope that a one-stage operation can be performed. This may not always be helpful. Davis et al reviewed 218 patients with DCIS. Of whom 64 did not have a preoperative MRI, and154 who did. ${ }^{26}$ Re-excision rates were similar (34\% and $39 \%$ respectively). Conversion rates to mastectomy were $9 \%$ and 8 $\%$. Average specimen weights at definitive surgery were $50 \mathrm{~g}$ and $49 \mathrm{~g}$ respectively. The authors concluded that preoperative MRI did not add benefit to the care of women with DCIS.

A group from the Academic Medical Center Amsterdam evaluated preoperative MRI to try and identify those patients with DCIS who are at high risk of invasive breast cancer. ${ }^{27}$ There were 125 with pure DCIS on core biopsy and 18 (14\%) had invasion on final histology. Suspicious enhancement on MRI was seen in 73 lesions, with a type 1 curve was seen in 12, type 2 in 19, and type 3 curve in 42 . The most predictive features on multivariate analysis for excluding invasive disease were no enhancement or a type 1 curve and this had a negative predictive value $98.5 \%$.

Pilewskie et al reviewed 352 patients with DCIS of whom 217 received MRI and 135 did not. ${ }^{28}$ There was no difference in terms of type of initial surgery and number of reoperations between the two groups. Successful breast conservation occurred more frequently in the no-MRI group. Additional biopsies were performed on $38 \%$ of the MRI group compared with $7 \%$ in the no-MRI group; $\geq 2$ additional biopsies were performed in $18 \%$ of the MRI group and $2 \%$ of the no-MRI group ( $\mathrm{p}<0.0001)$. These yielded a cancer diagnosis in $26 \%$ of MRI and $33 \%$ of no-MRI patients. MRI was not as good as mammography in detecting size of DCIS lesions preoperatively $52 \%$ of mammograms were accurate within $10 \mathrm{~mm}$ in $52 \%$ of cases compared with $41 \%$ of MRIs.

These data suggest that MRI is likely to pick up suspicious looking lesions which are histologically non-malignant and also does not, in most cases, add precision to determination of extent of DCIS. Hence MRI should not be used in a screening role when DCIS has been diagnosed but be reserved for those cases where there is genuine difficulty in identifying multicentric or more extensive disease. 
TABLE 2: Breast events in NSABP B24 and UK/ANZ DCIS trials.

\begin{tabular}{|c|c|c|c|c|c|c|c|c|c|c|c|}
\hline \multirow{2}{*}{$\begin{array}{l}\text { Feature } \\
\text { Treatment }\end{array}$} & \multirow[t]{2}{*}{$i$} & \multicolumn{3}{|c|}{ NSABP 24} & \multirow{2}{*}{$\begin{array}{l}1 \\
1 \\
1 \\
1\end{array}$} & \multicolumn{6}{|c|}{ UK/ANZ DCIS } \\
\hline & & Placebo & 1 & Tam & & Obs & Tam & 1 & $\mathrm{RT}$ & 1 & $\mathrm{Tam}+\mathrm{RT}$ \\
\hline Number & 1 & 900 & 7 & 899 & T & 544 & 567 & 1 & 267 & 1 & 376 \\
\hline Ipsilateral DCIS & i & $68(7.5 \%)$ & 1 & $60(6.7 \%)$ & ! & $96(18 \%)$ & $72(13 \%)$ & I & $16(6 \%)$ & i & $13(4 \%)$ \\
\hline Ipsilateral INV & I & $81(9 \%)$ & I & $59(6.5 \%)$ & I & $52(10 \%)$ & $49(9 \%)$ & I & $10(4 \%)$ & $i$ & $11(3 \%)$ \\
\hline Contralateral DCIS & 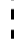 & $25(3 \%)$ & 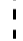 & $14(1.5 \%)$ & i & $9(2 \%)$ & $4(1 \%)$ & $i$ & $2(1 \%)$ & 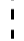 & $2(1 \%)$ \\
\hline Contralateral INV & 1 & $48(5 \%)$ & 1 & $30(3 \%)$ & 1 & $20(4 \%)$ & $7(1 \%)$ & 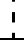 & $5(2 \%)$ & 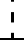 & $7(2 \%)$ \\
\hline
\end{tabular}

Abbreviations: Tam = tamoxifen; Obs = Observation; RT = Radio therapy

\section{Surgery}

Although a small proportion of patients with DCIS present with symptomatic disease, a lump or nipple discharge, the majority of cases will be picked up following mammography. When micro calcification graded as M3, M4 or M5 is observed an ultrasound will be carried out to determine whether there is an associated mass and also to examine whether abnormal axillary nodes are present. In the absence of a mass, a stereotactic core is performed and a marker inserted at the site of the core biopsies. This obviates need for a subsequent stereotactic core since the marker acts as a target for an ultrasound guided wire localisation. In some cases the DCIS will be clearly identified and extent can be measured. If there is extensive micro calcification, by taking cores from close to the margins may be useful so that there is histological proof of extent. This may help to persuade the patient to undergo mastectomy rather than breast conserving surgery.

\section{Localised wide excision}

The standard method of dealing with an impalpable breast lesion was wire localisation under ultrasound or stereotactic guidance, followed by surgical excision under general anaesthesia. ${ }^{29}$ Kohler et al evaluated ultrasound and mammography guided wire marking in 668 women with 741 impalpable breast lesions. Ultrasound was used in 418, mammography in 284 and for 39 lesions both techniques were combined. Specimen ultrasound suggested that $91 \%$ of lesions were resected completely and specimen $\mathrm{x}$-ray suggested complete resection in $89 \%$. Histologically $20 \%$ of the malignant lesions marked with sonography and $37 \%$ of cancers marked mammographically had involved margins. Re-excision was necessary in 10 patients localised by ultrasound and in 25 patients who were $\mathrm{x}$-ray localised.

Wire guided localisation (WGL) was usually successful but could be painful for the patient and occasionally the marker moved due to traction on the wire. To avoid this, in 1999, Luini et al. reported a new technique using a small quantity of $99 \mathrm{mTc}$-labelled colloidal albumin is injected directly into the lesion under stereotactic-radiographic or ultrasonic guidance. ${ }^{30} \mathrm{~A}$ gamma probe was then used to locate the lesion and guide the surgical excision. The results of radio-guided occult lesion localisation (ROLL) in 30 patients were compared with wire localisation in another 30 patients. In the WGL group, the mean distance from the lesion centre to the specimen margin was $25 \mathrm{~mm}$ compared with $14 \mathrm{~mm}$ in the radio-guided group so that ROLL removal reduced excision volume.

Several relatively small trials suggested that ROLL and WGL were equivalent. ${ }^{31,32,33}$ Van der Ploeg et al. reviewed the available literature and concluded that ROLL was a promising technique, which achieved complete excision more frequently than WGL and was more accurate and faster. ${ }^{34}$ Subsequently, contrary evidence came from a multicentre randomised controlled trial comparing ROLL and WGL for preoperative tumour localisation. ${ }^{35}$ Complete tumour removal was achieved in 140/162 (86 \%) patients in the ROLL group and 134/152 (88 \%) patients in the WGL group. Re-excision was required in $12 \%$ of the ROLL group versus $10 \%$ in the WGL group. Mean specimen volumes were $71 \mathrm{~cm}^{3}$ versus $64 \mathrm{~cm}^{3}$ in the ROLL and WGL arms respectively. No significant differences were observed in the duration and difficulty of either the radiological or surgical procedures. It was suggested that ROLL cannot replace WGL as the standard of care.

Arentz et al hypothesized that the hematoma-directed ultrasound-guided (HUG) procedure to localise impalpable lesions would allow excision without the problems of WGL and decrease the high rate of margin positivity. ${ }^{36} \mathrm{~A}$ multifrequency linear array transducer was employed intraoperatively for the HUG localisation, and a block of tissue surrounding the hematoma removed. Results were reported in 455 patients of whom 126 (28 \%) had needle localization and 329 (72 \%) underwent HUG. The previous core-biopsy site was successfully excised in all patients using HUG. Of those with cancer margins were positive in $24 \%$ of HUG compared with $47 \%$ of those needle localised. This suggests that HUG is more accurate in localising impalpable lesions than WGL but the technique is dependent upon the surgeon being trained in this aspect of ultrasound.

\section{Margins}

Although there is agreement that the aim is to achieve uninvolved margins, there has been disagreement concerning margin width. Dunne et al carried out a review of published trials examining outcomes after breast conserving surgery and 
radiotherapy for DCIS. ${ }^{37}$ There were 4,660 patients and those with negative margins were at substantially reduced risk of relapse compared with those with positive margins (Odds ratio 0.36$)$. Negative margins were better than close margins in terms of relapse (OR 0.59). A $2 \mathrm{~mm}$ margin was better than $<2 \mathrm{~mm}$ (OR 0.53). There was however no significant difference in relapse rate with margins $2-5 \mathrm{~mm}$ or $>5 \mathrm{~mm}$.

Wang et al criticised the methodology used by Dunne, arguing that only randomised trials were included and here was duplication of data with multiple studies from the same institution drawing on overlapping patient sources. ${ }^{38}$ Additionally, Dunne pooled study results and did not use them as a stratification factor. Wang et al identified 21 randomised and non-randomised studies with a total of 7564 women and performed a network meta-analysis. Of the patients, 3098 were treated by BCS alone and 4466 with additional radiotherapy. Ipsilateral relapse occurred in 565 (16\%) and 501 (11 $\%)$ respectively.

Comparing negative with positive margins, there was a reduced relapse risk both with radiotherapy $(\mathrm{OR}=0.46)$, and without radiotherapy $(\mathrm{OR}=0.34)$. For positive margins versus negative margin $>0 \mathrm{~mm},>2,>5$ and $\geq 10 \mathrm{~mm}$ the ORs were $0.45,0.38,0.55$, and 0.17 respectively. When comparing a negative margin $>2 \mathrm{~mm}$, with a negative margin of $\geq 10 \mathrm{~mm}$ the latter carried a lower risk of relapse $(O R=0.46)$. They concluded that within the constraints of satisfactory cosmesis, an attempt should be made to achieve wide negative margins but that further studies should determine whether margin thresholds $>10 \mathrm{~mm}$ are of benefit.

\section{Sentinel node biopsy}

Ansari et al conducted a meta-analysis of results from 22 series of sentinel biopsy (SNB) in 3166 patients with a pre-operative diagnosis of DCIS. ${ }^{39}$ The average rate of sentinel node involvement was approximately $7 \%$. When however the post-operative diagnosis was DCIS alone the rate of nodal positivity fell to $4 \%$. Studies predicting invasive disease after a core biopsy showing DCIS have shown that the significant variables were high grade DCIS, $>2.5 \mathrm{~cm},<12$ core biopsies, palpable mass, mass on imaging and inflammatory infiltrate in the core biopsy.

Osako et al investigated SNB in a large cohort of women with DCIS. The node was subjected to frozen section (FS) analysis in 338 patients treated between 2007 and 2009. For 285 who undergoing surgery between 2009 and 2011 the sentinel node (SN) was examined using the one-step nucleic acid amplification (OSNA) assay. More cases of SN metastases were detected by OSNA than FS (12/285 (4 \% versus 1/338 $(0.3 \%)$. The majority were micrometastases. The characteristics of DCIS at high-risk of invasion (mass, size, grade, and comedo type, preoperative breast biopsy) did not apply to OSNA assay-positive DCIS.
For those patients having immediate reconstruction it is best that the SNB is carried out as a stand-alone procedure in order that the axillary nodal status in known before embarking on what may be a complex reconstruction. If this has not been carried out the need to carry out carry out a completion axillary clearance may compromise the achievement of a good cosmetic result.

\section{Which patients with DCIS require a mastectomy?}

There is probably less argument about indications for mastectomy in women with DCIS than there is concerning suitability for breast conserving surgery. An absolute indication is histologically proven presence of DCIS in more than one quadrant. In the event of multifocal rather than multicentric disease, the main consideration is whether the DCIS can be extirpated and still leaves the patient with a reasonable cosmetic result. Trials have varied in maximum allowable extent of DCIS but the clinical decision should be based on the extent of disease in relation to the size of the breast.

When the extent of DCIS mandates mastectomy a sentinel node biopsy should be performed. Upstaging from DCIS to invasive disease occurs in up to $20 \%$ of cases with pure DCIS on the original core. ${ }^{40,41}$ When invasive disease is present it is now accepted that; provided axillary ultrasound is normal, sentinel node biopsy is the best method of staging the axilla. ${ }^{42}$ Ansari et al reported a meta-analysis of results from 22 series of sentinel biopsy in 3166 patients with a pre-operative diagnosis of DCIS. ${ }^{43}$ The average rate of sentinel node involvement was approximately $7 \%$. When however the post-operative diagnosis was DCIS alone the rate of nodal positivity fell to $3.7 \%$.

The greater the extent of DCIS the higher the risk of associated invasion. In a series of 398 women with pure DCIS on core biopsy operated on at the MD Anderson Hospital $20 \%$ were upgraded on final histology. ${ }^{44}$ Multivariate analysis indicated that the significant prognostic variables included younger age, high grade DCIS and mammographic size $\geq 4 \mathrm{~cm}$. The same group reported later that invasive carcinoma was found at definitive surgery in $30 \%$ of those with DCIS $>15 \mathrm{~mm}$ and only $11 \%$ of those with DCIS $\leq 15 \mathrm{~mm} .{ }^{45}$

It is often assumed that a total mastectomy provides a cure for DCIS, with a zero risk of local recurrence. Unfortunately this is not true. Residual breast tissue may be left behind, particularly in the axillary tail and in the most inferior part of the breast. The larger series that have reported recurrence rates after mastectomy for ductal carcinoma. In situ are summarised in Table 3. ${ }^{46,47,48,49}$ This indicates that 1-2\% of patients will develop a relapse. Kelley et al used the USC Van Nuys Prognostic Index, which is an algorithm based on DCIS size, nuclear grade, necrosis, margin width, and patient age. Recurrence occurred in 11 patients, all of whom scored 10-12 
using the USC/VNPI and all had multifocal disease and comedo-type necrosis.

TABLE 3: Recurrence after mastectomy for DCIS.

\begin{tabular}{lccc}
\hline \hline Author & N & Recurrence & Follow-up \\
\hline Bedwani 1981 & 112 & $1(1 \%)$ & 60 months \\
Silverstein 1995 & 228 & $2(1 \%)$ & 78 months \\
Van der Velden 200748 & 408 & $4(1 \%)$ & 65 months \\
Kelley 201149 & 496 & $11(2 \%)$ & 83 months \\
\hline \hline
\end{tabular}

\section{Management plan}

An algorithm for management of DCIS is given in Figure 1. Treatment should not be planned until there is histological confirmation of the diagnosis of DCIS, accepting that up to 20 $\%$ of cases will eventually prove to have invasion/microinvasion. Outside of research protocols breast MRI should be confined to those cases where the extent of disease is indeterminate. All patients undergoing mastectomy for extensive DCIS should have a sentinel node biopsy as part of primary treatment. Sentinel node biopsy should also be considered for patients with DCIS $\geq 2.5 \mathrm{~cm}$ having a wide excision because of the high risk of invasive disease being present. ${ }^{39}$

If a mastectomy is deemed necessary this will normally be a skin sparing procedure since this has been shown to be as effective as a standard mastectomy in terms of local control of disease and can help to achieve a better cosmetic outcome. ${ }^{50}$ The Gustave Roussy Breast Cancer Study Group Data analysed 238 consecutive patients diagnosed with DCIS and who had undergone mastectomy and SNB between 2005 and $2011 .{ }^{51}$ Of these, $57 \%$ had immediate breast reconstruction 43 $\%$ did not. The commonest reason for immediate reconstruction not being offered was that it had not been mentioned by the surgeon $(33.4 \%)$. The rate of immediate reconstruction was highest in those $<50$ and (52.2\%), and was lower among women with diabetes $(0.7 \%)$ or obesity $(8.8 \%)$. The choice of reconstruction was unaffected by tobacco use or positive lymph node status. This report from a centre of excellence highlights a potential lacuna in communication. Discussion of immediate reconstruction should be an intrinsic part of discussion between the surgeon and the patient with DCIS.

In a small series of 14 nipple-saving mastectomies (NSM), Nahabedian and Tsangaris reported that sensation was present in $43 \%$, delayed healing occurred in $29 \%$ and symmetry was achieved in five of 50\%. ${ }^{52}$ Local relapse occurred $27 \%$, and secondary procedures were required in 36\%. Reviewing the evidence for efficacy and safety of nipple-preserving mastectomy in 2006, Garcia-Etienne and Borgen concluded that the published studies lacked the power to determine a role for NSM..$^{53}$

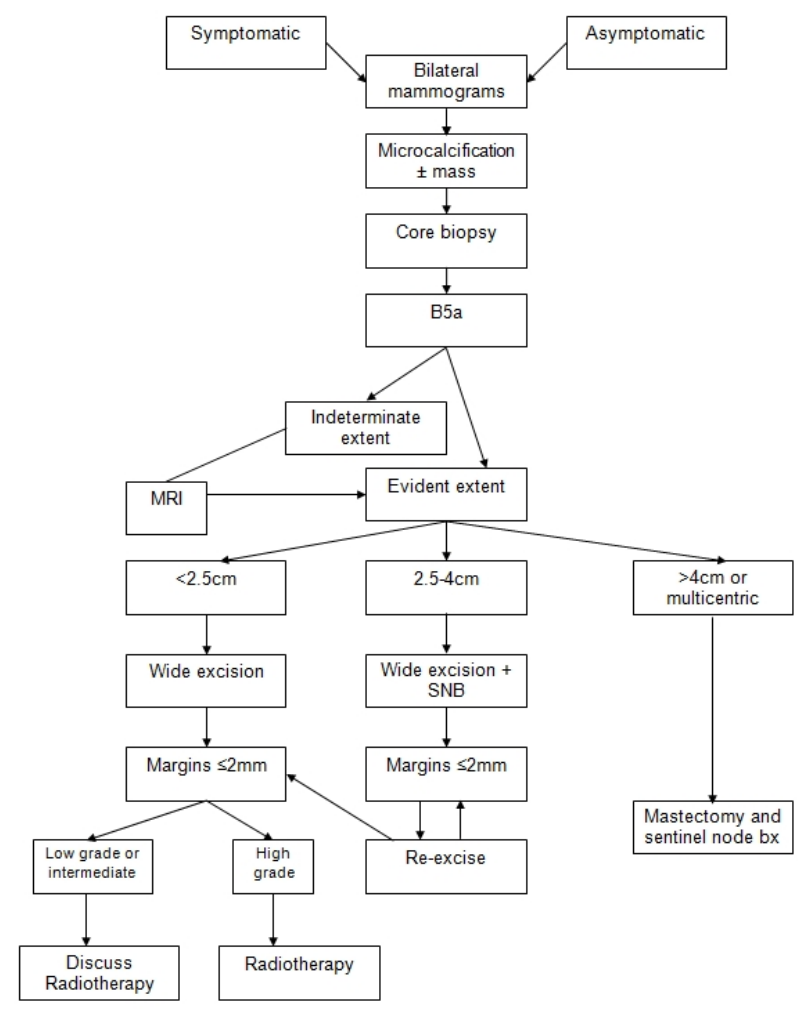

FIG. 1: Algorithms for DCIS management.

The situation changed when Petit et al reported a series of 570 patients with breast cancer treated by NSM were performed for carcinoma. ${ }^{54}$ An immediate frozen section was taken from retroareolar tissue. If tumour was present the patient was not treated by NSM (63/570, 12\%). Likelihood of areola positive histology increased with tumour size but was unrelated to the nodal status. The local recurrence rate was $0.9 \%$ per year. Provided that appropriate control is observed and in experienced hands, NPM is a safe surgical option in selected patients with DCIS.

\section{Conflict of interest}

The authors declare that they have no conflicts of interest. The authors alone are responsible for the content and writing of the paper.

\section{References}

1. Veronesi U, Saccozzi R, Del Vecchio M, et al. Comparing radical mastectomy with quadrantectomy, axillary dissection, and radiotherapy in patients with small cancers of the breast. NEngl J Med 1981; 305: 6-11.

2. Fisher B, Redmond C, Poisson R, et al. Eight-year results of a randomized clinical trial comparing total mastectomy and lumpectomy with or without 
irradiation in the treatment of breast cancer. $N$ Engl JMed 1989; 320: 822-8.

3. Van Dongen JA, Bartelink H, Fentiman IS, et al. Factors influencing local relapse and survival and results of salvage treatment after breast-conserving therapy in operable breast cancer: EORTC Trial 10801, breast conservation compared with mastectomy in TNM stage I and II breast cancer. Eur $J$ Cancer 1992; 28A: 801-5.

4. Jacobson JA, Danforth DN, Cowan KH, et al. Ten-year results of a comparison of conservation with mastectomy in the treatment of stage I and II breast cancer. N Engl J Med 1995; 332: 907-11.

5. van Dongen JA, Bartelink H, Fentiman IS, Peterse JL. Ductal carcinoma in situ (DCIS) of the breast -a therapeutic dilemma. Eur J Surg Oncol 1987; 13: 123-126.

6. Virnig BA, Tuttle TM, Shamliyan T, Kane RL. Ductal carcinoma in situ of the breast: a systematic review of incidence, treatment, and outcomes. $J$ Natl Cancer Inst 2010; 102:170-178.

7. Pinder SE, Duggan C, Ellis IO, Cuzick J, Forbes JF, Bishop H, Fentiman IS \& George WD. A new pathological system for grading DCIS with improved prediction of local recurrence: results from the UKCCCR/ANZ DCIS trial. Br J Cancer 2010; 103: 94-100.

8. Bijker N, Petersee JL, Duchateau L, Julien J-P, Fentiman IS, et al. Risk factors for recurrence and metastasis after breast conserving therapy for ductal carcinoma in situ: analysis of European Organisation for Research and Treatment of cancer trial 10853. J Clin Oncol 2001; 19: 2263-2271.

9. Esserman L, Shieh Y, Thompson I. Rethinking screening for breast cancer and prostate cancer. JAMA 2009; 302:1685-1692.

10. Solin LJ, Gray R, Baehner FL, Butler SM, Hughes $\mathrm{LL}$, et al. A multigene expression assay to predict local recurrence risk for ductal carcinoma in situ of the breast. J Natl Cancer Inst 2013; 105:701-710.

11. Wapnir IL, Dignam JJ, Fisher B, Mamounas EP, Stewart J. Anderson SJ, et al. Long-term outcomes of invasive ipsilateral breast tumor recurrences after lumpectomy in NSABP B-17 and B-24 randomized clinical trials for DCIS. J Natl Cancer Inst 2011; 103: 478-488.

12. Cuzick J, Sestak I, Pinder SE, Ellis IO, Forsyth S, Bundred NJ, Forbes JF, Bishop H, Fentiman IS, George WD. Effect of tamoxifen and radiotherapy in women with locally excised ductal carcinoma in situ: long-term results from the UK/ANZ DCIS trial. Lancet Oncol 2011; 12: 21-29.

13. Donker M, Litiere S, Werutsky G, Julien JP, Fentiman IS, et al. Breast-conserving treatment with or without radiotherapy in Ductal Carcinoma In Situ: 15-year Recurrence Rates and Outcome
After a Recurrence, from the EORTC 10853 Randomized Phase III Trial. Journal of Clinical Oncology 2013; 31:4054-4059.

14. Holmberg L, Garmo H, Granstrand B, et al. Absolute risk reductions for local recurrence after postoperative radiotherapy after sector resection for ductal carcinoma in situ of the breast. J Clin Oncol 2008; 26:1247-1252.

15. Correa C, McGale P, Taylor C, et al. Early Breast Cancer Trialists' Collaborative Group. J NatlCancer Inst Monogr 2010; 2010:162-177.

16. Wehner P, Lagios MD, Silverstein MJ. DCIS treated with excision alone using the national Comprehensive Cancer Network (NCCN) Guidelines. Ann Surg Oncol 2013; 20: 3175-9.

17. Fisher B, Dignam J, Wolmark N, et al. Tamoxifen in treatment of intraductal breast cancer: National Surgical Adjuvant Breast and Bowel Project B-24 randomised controlled trial. Lancet 1999; 353: 1993-2000.

18. Houghton J, George WD, Cuzick J, Duggan C, Fentiman IS, Spittle M; UK Coordinating Committee on Cancer Research; Ductal Carcinoma in situ Working Party; DCIS trialists in the UK, Australia, and New Zealand. Radiotherapy and tamoxifen in women with completely excised ductal carcinoma in situ of the breast in the UK, Australia, and New Zealand: randomised controlled trial. Lancet 2003; 362:95-102.

19. Wapnir IL, Dignam JJ, Fisher B, et al. Long-term outcomes of invasive ipsilateral breast tumor recurrences after lumpectomy in NSABP B-17 and B-24 randomized clinical trials for DCIS. J Natl Cancer Inst 2011; 103:478-488.

20. Allred DC, Anderson SJ, Paik S, et al. Adjuvant tamoxifen reduces subsequent breast cancer in women with estrogen receptor-positive ductal carcinoma in situ: a study based on NSABP Protocol B-24. J Clin Oncol 2012; 30:1268-1273.

21. Cuzick J, Sestak I, Pinder SE, et al. Effect of tamoxifen and radiotherapy in women with locally excised ductal carcinoma in situ: long-term results from the UK/ANZ DCIS trial. Lancet Oncol 2011; 12: 21-9.

22. StaleyH, McCallum I, Bruce J. Postoperative tamoxifen for ductal carcinoma in situ. Cochrane Database Syst Rev 2012; 10:CD007847.

23. Cuzick J. Aromatase inhibitors in prevention - data from the ATAC (Arimidex, tamoxifen alone or in combination) trial and the design of IBIS-II (the second International Breast Cancer Intervention Study). Recent Results Cancer Res 2003; 163:96-103.

24. Dixon JM, Faratian D, White S et al. DCIS and aromatase inhibitors. I Steroid Biochem Mol Biol 2007; 106:173-9. 
25. Chen YY, DeVries S, Anderson J, Lessing J, et al. Pathologic and biologic response to preoperative endocrine therapy in patients with ER-positive ductal carcinoma in situ. BMC Cancer 2009; 9:285.

26. Davis KL, Barth RJ Jr, Gui J, et al. Use of MRI in preoperative planning for women with newly diagnosed DCIS: risk or benefit? Ann Surg Oncol 2012; 19:3270-4.

27. Deurloo EE, Sriram JD, Teertstra HJ, et al. MRI of the breast in patients with DCIS to exclude the presence of invasive disease. Eur Radiol 2012; 22:1504-11.

28. Pilewskie M, Kennedy C, Shappell C, et al. Effect of MRI on the management of ductal carcinoma in situ of the breast. Ann Surg Oncol 2013; 20:1522-9.

29. Chaudary MA, Reidy JF, Chaudhuri R, Millis RR, Hayward JL, Fentiman IS. A new and improved device for the pre-operative localisation of impalpable breast lesions. Br J Surgery 1990; 77: 1191-1192.

30. Luini A, Zurrida S, Paganelli G, et al. Comparison of radioguided excision with wire localization of occult breast lesions. Br J Surg 1999; 86:522-5.

31. Medina-Franco H, Abarca-Perez L, García-Alvarez MN. Radioguided occult lesion localization (ROLL) versus wire-guided lumpectomy for non-palpable breast lesions: a randomized prospective evaluation. J Surg Oncol 2008; 97:108-11.

32. Mariscal Martínez AM, Sola M, Perez de Tudela A. Nonpalpable breast cancer lesions: randomized comparison with wire localization in patients undergoing conservative surgery and sentinel node biopsy. AJR 2009; 193:1001-1009.

33. Sarlos D, Frey LD, Haueisen H, Landmann G, Kots LA, Schaer G. Radioguided occult lesion localization (ROLL) for treatment and diagnosis of malignant and premalignant breast lesions combined with sentinel node biopsy: a prospective clinical trial with 100 patients. Eur J Surg Oncol 2009; 35:403-8.

34. van der Ploeg IM, Hobbelink M, van den Bosch MA. Radioguided occult lesion localisation' (ROLL) for non-palpable breast lesions: a review of the relevant literature. Eur J Surg Oncol 2008; 34:1-5.

35. Postma EL, Verkooijen HM, van Esser S, Hobbelink MG et al, Efficacy of 'radioguided occult lesion localisation' (ROLL) versus 'wire-guided localisation' (WGL) in breast conserving surgery for non-palpable breast cancer: a randomised controlled multicentre trial. Breast Cancer Res Treat 2012; 136:469-78.

36. Arentz C, Baxter K, Boneti C, et al. Ten-year experience with hematoma-directed ultrasound-guided (HUG) breast lumpectomy. Ann Surg Oncol 2010; 17 Suppl 3:378-83.
37. Dunne C, Burke JP, Morrow M, Kell MR. Effect of margin status on local recurrence after breast conservation and radiation therapy for ductal carcinoma in situ. JClin Oncol 2009; 27:1615-1620.

38. Wang S-Y, Chu H, Shamliyan T, et al. Network meta-analysis of margin threshold for women with ductal carcinoma in situ. J Natl Cancer Inst 2012; 104:507-516.

39. Ansari B, Ogston SA, Purdie CA, et al. Meta-analysis of sentinel node biopsy in ductal carcinoma in situ of the breast. Br J Surg 2008; 95: 547-554.

40. Sakr R, Antoine M, Barranger E, et al. Value of sentinel lymph node biopsy in breast ductal carcinoma in situ upstaged to invasive carcinoma. Breast J2008; 14: 55-60.

41. Van la Parra RF, Ernst MF, Barneveld PC, et al. The value of sentinel lymph node biopsy in breast ductal carcinoma in situ (DCIS) and DCIS with microinvasion of the breast. Eur J Surg Oncol 2008; 34: 631-5.

42. Veronesi U, Paganelli G, Viale G, Luini A, Zurrida $\mathrm{S}$, Galimberti V et al. A randomized comparison of sentinel-node biopsy with routine axillary dissection in breastcancer. $N$ Engl JMed 2003; 349: 546-553.

43. Ansari B, Ogston SA, Purdie CA, et al. Meta-analysis of sentinel node biopsy in ductal carcinoma in situ of the breast. Br J Surg 2008; 95: 547-554.

44. Yen TWE, Hunt KK, Ross MI, et al. Predictors of invasive breast cancer in patients with an initial diagnosis of ductal carcinoma in situ: a guide to selective use of sentinel lymph node biopsy in management of ductal carcinoma in situ. J Am Coll Surg 2005; 200: 516-526.

45. Huo L, Sneige N, Hunt KK, et al. Predictors of invasion in patients with core-needle biopsy-diagnosed ductal carcinoma in situ and recommendations for a selective approach to sentinel node biopsy in ductal carcinoma in situ. Cancer 2006; 107: 1760-8.

46. Bedwani R, Vana J, Rosner D, et al. Management and survival of female patients with "minimal" breast cancer. Cancer 1981; 47: 2769-2778.

47. Silverstein MJ, Barth A, Poller DN, et al. Ten-year results comparing mastectomy to excision and radiation therapy for ductal carcinoma in situ of the breast. Eur J Cancer 1995; 31:1425-1427.

48. Schouten van der Velden AP, van Vugt R, Van Dijck JA, et al. Local recurrences after different treatment strategies for ductal carcinoma in situ of the breast: a population-based study in the East Netherlands. Int J Radiation Oncology Biol Phys 2007; 69:703-10. 
49. Kelley L, Silverstein M, Guerra L. Analyzing the risk of recurrence after mastectomy for DCIS: a new use for the USC/Van Nuys Prognostic Index. Ann Surg Oncol 2011; 18:459-462.

50. Kroll SS, Schusterman MA, Tadjalli HE, Singletary SE, Ames FC. Risk of recurrence after treatment of early breast cancer with skin-sparing mastectomy. Ann Surg Oncol 1997; 4:193-7.

51. Naoura I, Mazouni C, Ghanimeh J, Leymarie N, et al. Factors influencing the decision to offer immediate breast reconstruction after mastectomy for ductal carcinoma in situ (DCIS): the Institut Gustave Roussy Breast Cancer Study Group experience. Breast 2013; 22:673-5.
52. Nahabedian MY, Tsangaris TN Breast reconstruction following subcutaneous mastectomy for cancer: a critical appraisal of the nipple-areola complex. Plast Reconstr Surg 2006; 117:1083-90.

53. Garcia-Etienne CA, Borgen PI. Update on the indications for nipple-sparing mastectomy. J Support Oncol 2006; 4:225-30.

54. Petit JY, Veronesi U, Rey P, et al. Nipple-sparing mastectomy: risk of nipple-areolar recurrences in a series of 579 cases. Breast Cancer Res Treat 2009; 114:97-101. 